\title{
INDEX OF NAMES
}

Abendroth, W., 318.

Abernethy, G. L., 516 .

Ábrányi, A., 344.

Action sociale et service social, 162 .

Adler, S., $47^{8}$.

Agrarian Societies in Transition, $461 / 462$.

Albania, 322.

Albers, W., 488 .

Albertini, J. M., 326.

Alembert, J. d', $323 / 324 / 325$

Alexander, R. J., 467.

Alexandrov, V., 5 ro.

Allemann, F. R., 166/167.

Allen, G., 506.

Allen, H. B., 462.

Allen, V. L., 503.

Alphen de Veer, M. R. v., I 28.

Alquié, F., 292.

Alyea, B. R., $468 / 469$.

Alyea, P. E., $468 / 469$.

American Labor Union

Periodicals, I 42.

American Radicals, 3 II.

Andersson, I., I 80 .

Andreas, W., 157/158.

Andrews, J. C., 144 .

Andronikof, C., 512.

Angell, R. C., 303.

Anger, W., 494.

Ankenbrank, K., 173.

Anshen, M., 469.

Antelme, R., 494 .

Aptheker, H., 469 .

The Arabs in Israel (publ. Baade, F., 137, 318.

1952), 516.
The Arabs in Israel (publ.

I955), 156 .

Aranyossi, M., 458 .

Arbeiterjugend gestern und heute, 167.

Das Arbeitsrecht in der D.D.R., 516.

Archer, P., 5 18.

Archivar und Historiker, 494.

Arensberg, C. M., 293, 302.

Ariès, Ph., 133.

Armand, E., 288.

Armand, F., 323/324/325.

Armee gegen die Freiheit, 328/329.

Arndt, E., 45 I.

Arnold, G. L., $47^{6}$.

Arnold, H., 329.

Arnold, K., 332.

Aron, R., 303, 462.

Ashley, M., 503 .

Ashton, E. T., I76.

Aspects of Human Equality, 288.

Aspects sociaux de l'industrialisation et de l'urbanisation en Afrique au sud du Sahara, I 4I.

Les attitudes des mineurs du Centre-Midi et l'évolution de l'emploi, 488.

Aufgaben deutscher Forschung, 167 .

Aulas, B., 156 .

Babeuf, G., 323/324/325.
Baboulène, J., 326.

Bach, E., 336 .

Badajew, A., 346.

Bäuerliche Familienbetriebe in Nordrhein-Westfalen, 494/495.

Balogh, J. K., 516.

Bancroft, F., 470.

Banning, W., 288.

Baran, P. A., 293.

Baranès, A., I62.

Baratz, J., 319.

Barber, B., $451 / 452$.

Barents, J., I 34 .

Barghoorn, F. C., I 80.

Barjonet, M., 323/324/325.

Barker, E., 46r.

Baron, H., 46r.

Barraclough, G., 133.

Bartoli, H., 452.

Barton, P., 321 .

Bassenge, F., 291.

Bastin, J., 319.

Bates, M., I 29/1 30 .

Battistini, L. H., x 52, 320.

Baudin, L., I42, 295.

Bauer, A., 335/336.

Bauer, R. A., 5 Io.

Baumont, M., 323.

Beardsley, S. W., 516 .

Beaucourt, Ch., 484/485.

Beaumarchais, P. A. C. de, $323 / 324 / 325$.

Beauvoir, S. de, 478 .

Begin, M., $510 / 511$.

Beiträge zum neuen $\mathrm{Ge}$ schichtsbild, 459.

Beiträge zur russischen, pol- 
nischen und deutschen Boudot, F., 323.

Geschichte, 32r.

Bell, N. W., 468.

Bellah, R. N., 320.

Bellamy, R. F., 457.

Beloff, M., 490.

Beltow, N., see G. W. Plekhanov.

Benham, F., 152.

Bennett, S. V., 3 II.

Benoist, A., I63.

Benson, L., 293.

Beqiraj, M., 322.

Berger, A., 326.

Bergmann, Th., 516 .

Berkes, N., 315 .

Berkman, P. L., 485 .

Bernard, J., 303.

Berque, J., 465 .

Berton, P., 346.

Die berufliche und soziale

Gliederung der Bevölkerung der Bundesrepublik, 167.

Die Beschäftigungsfreiheit der Montanfacharbeiter, I 8 .

Bessie, A., 3 Ir.

Beutin, L., I67.

Beyer, H., 495 .

Bezemer, J. W., I80.

Bicrbaum, M., 329.

Bihalji-Merin, L., I 86.

Bihalji-Merin, O., 186.

Bismarck, K. v., 26.

Black, C. E., 180 .

Blanqui, A., $323 / 324 / 325$.

Bleton, P., I 28

Bloch, E., 288/289, 28 9.

Bloch, M., 488/489.

Bobrowski, C., 18 I.

Boelcke, W., 499.

Boer, H. A. de, 462 .

Boettcher, E., 348.

Bohn, H., 328/329.

Borchert, H., 329 .

Borcke-Stargordt, H., 495 .

Bornkamm, G., I 26.

Bosshard, W., I 2.

Bossy, G., 322.

Bot, P. N. M., 177 .

Bottigelli, É., I $58,32 \mathrm{~T}$.
Bourne, R., 469 .

Bouvier-Ajam, M., 489 .

Bowen, H., 459/460.

Bowles, W. D., I 82.

Boyer, R. O., 469/470.

Bracher, K. D., 329.

Brackel, P. O. M., 509.

Brand, J., 486.

Brandt, L., I 67, 516.

Braun, H., $329 / 330$.

Braunthal, J., 516.

Brautigam, J., I 77 .

Bray, J. F., I 34.

Bremme, G., 330.

Brepohl, W., 495.

Breuvery, E. S. de, I 4 o.

Brimmell, J. H., 516.

Brochon, P., $323 / 324 / 325$.

Brock, P., 487/488.

Brogan, D. W., 323.

Bromage, M. C., I62.

Brühl, F., 335 .

Brunner, O., 299.

Bruijning, C. F. A., 310.

Bryson, L., 288.

Brzezinski, Z. K., 453.

Buber-Neumann, M., 495/ 496.

Buck, E. H., I44.

Buck, H., 499.

Buck, S. J., I44.

Buonarroti, Ph., 323/324/ 325.

Burns, E. M., 452.

Burns, J. M., 470.

Bychowski, B. E., 290.

Caillot, R., 326.

Calcat, R., 326.

Calvez, J. Y., 289/290, 484/ 485,5 II.

Le campagne emiliane nell' epoca moderna, 507.

Campbell, W. K. H., 302/ 303.

Captive Rumania, 179.

Carter, P. A., I44/145.

Carticr-Bresson, H., I 54.

Catz, E. W., 516.

Celier, L., I63.

A Century of Family Law,
$173 / 174$.

Chambelland, C., $\mathrm{I}_{3}$.

La chanson française, 323 / $324 / 325$.

Charbonnel, P., 323/324/ 325 .

Chardonnet, J., 307.

Chase, H. V., 457.

China. List of References, si6.

China in Transition, 154.

Chinese Social History, 478.

Chirovsky, N. L. F., 346.

Christen oder Bolschewisten, I 26.

Christendom en nationalisme, 134 .

Cirtautas, K. C., 452.

Claudius, W., i 84.

Cobban, A., $46 \mathrm{r}$.

Codignola, T., 507.

Cogley, J., I45.

Cogniot, G., 181, 323/324/ 325.

Cohen, D., 177.

Cohn, D. L., I45, 311 .

Cohn, N., 459.

Cole, G. D. H., 303.

Coleman, J. S., 148.

Colliard, C. A., 326.

Colm, G., 140.

Combe, P., 489 .

Common Frontiers of the Social Sciences, 293.

$\mathrm{XXe}$ Congrès du Parti Communiste de l'Union Soviétique, $18 \mathrm{I}$.

Contemporary China, 478/ 479.

Contemporary Collective

Bargaining in Seven Countries, 303 .

Contributions to Indian Sociology, 516 .

Conze, W., 496, 516 .

Cooke, J. E., 470.

Cordelier, S., 325 .

Cornevin, R., 465 .

Cornforth, M., 290.

Cornuau, Ph., 488.

Coudenhove-Kalergi, R., $462 / 463$. 


\author{
Coumil, P., 326. \\ Cox, S., gi6. \\ Crane, F. R., I73/r 74 . \\ Cranston, M., 338/339. \\ Cretzianu, A., 179. \\ Cropsey, J., $45^{2}$. \\ Crosland, C. A. R., 339 . \\ Culture ouvrière et action \\ syndicale, 325 . \\ Current Soviet Policies, 5 II . \\ Cuvillier, A., 325, 455 .
}

Dahrendorf, R., 453, 5I6.

Dale, L. A., 489.

Dallin, A., 484 .

Dalton, H., 503.

Daniel, J., 449.

Daniels, R. V., 514.

Darby, H. C., 46 I.

Dautry, J., 323/324/325, 325.

David, H., 293.

David, M., 485 .

Davis, K., 46I/462.

Dawson, Chr., 158 .

De la nature des conflits, 303.

Debs, G., I 48.

Delprat, R., 304.

Demographic Yearbook 1956, 463.

Denis, H., 5 I 7 .

Denkers van deze tijd, 293.

Dennis, E., I45.

Dennis, P., I45.

Des Accords, M., 49 I.

Descartes, R., 323/324/325.

Descola, J., 459.

A Design for Democracy, I 74 .

Desné, R., 323/324/325.

Detraz, A., 326.

Développement d'une classe moyenne dans les pays tropicaux.., 137.

Dib, G. M., I 52.

Diderot, D., $323 / 324 / 325$.

Die, B. C. de, 178,517 .

Dietze, W., 496.

Dimitroff, G., $16 \mathrm{r}$.

Dixon, J. G., 300/301.

Dohne, E., 494/495.
Dommanget, M., I63.

Dorfuntersuchungen, 517 .

Dornemann, L., 496.

Drake, H., 336 .

Draper, Th., 470.

Drogat, N., $303 / 304$.

Droz, J., 158.

Dru, A., 325 .

Dubreuil, H., 134, 301 .

Dufay, F., i54.

Dulles, F. R., 470/47I.

Dumbauld, E., 471 .

Dumont, L., 480, 516.

Duncan, O. D., r31, r45/ 146.

Dunchi, N., 344.

Dunlop, J. T., 298, $456 / 457$.

Duroselle, J. B., 295, 484/ 485.

Du Rostu, J., 49 I.

Dutch Immigrant Memoirs and Related Writings, I 46 .

Duveau, G., $4^{89}$.

Duverger, M., 490.

Dijk, R. v., 126.

Économie et civilisation, 304.

Edgell, A. G., 5 I6.

Eells, W. C., 137.

Egretaud, M., 517.

Egypt and the Suez Canal, 517.

Eichler, H., 294.

Eichstädt, U., 487.

Eisermann, G., I67/I68.

Ekirch Jr., A. A., 146.

Eldersveld, S. J., I 3 I.

Les élections du 2 janvier $1956,490$.

Ellemers, J. E., $177 / 178$.

Elmer, M. C., 146.

Elwert, K., 497.

Engels, F., I58, 321, 330, $334,334 / 335,500$.

English Historical Documents, 339 .

Erben, B., 517 .

Erdmann, G., 330.

Erfurt, W., I68.

Erfurth, W., 33 I.
Ergebnisbeteiligung, $33 \mathrm{I}$.

Erickson, Ch., 47r.

Erkes, E., I 54 .

Erlenbach, 'Th. S., 517.

Esch, P. v. d., 459 .

Esperienze e studi socialisti, 507.

Essays on Liberty, 138 .

Esveld, N. E. H. v., 138 .

E-Tu Zen Sun, 478.

Eulau, H., 13 I.

Europa - Erbe und Aufgabe, 158.

European Jewry Ten Years after the War, $4^{84}$.

Evalenko, R., 322/323.

Les exigences humaines de l'expansion économique, 304 .

Fallers, L. A., 466.

Far Eastern Affairs, 476.

Faravelli, G., 507 .

Farman, C. H., 517 .

Farmer, B. H., 316/317.

The Fate of East Central Europe, $x 59$.

Faulkner, H. U., $47 x$.

Faure, P., 490.

Faust, C. H., 288.

Fedeli, U., 288.

Feibleman, J. K., I 28/ 229.

Fejtö, F., 343 .

Fellermeier, J., I 26.

Fernández, J., 509.

Fetscher, I., 1 26, 455 .

Feuerbach, L., 290.

Finkelstein, L., 288.

Fischer, G., 514 .

Fischer, R., I 38.

Fisher, L. H., 293.

Fiske, M., 293.

Fite, G. C., 475 .

Flavay, L., 326.

Fleisher, W., 345/346.

Fleming, D. F., 468.

Fleming, H, , 148/ 449 .

Floud, J. E., 339.

Folliet, J., 326.

Le fonctionnement des

entreprises nationalisées

en France, 326. 
Foner, Ph, S., $471 / 47^{2}$.

Footman, D., 184 .

Forde, D., I4I.

Fortes, M., I 30/I3I.

Fourier, Ch., 323/324/325.

Fourrier, Ch., 453 .

France: Government and Society, 490.

La France, 326.

France, A., 323/324/325.

France, R. W., 472 .

Francis, J. de, 478 .

Frandon, I. M., 490.

Frankel, Ch, 290.

Frankfurter, F., I46/I47.

Franz, G., 171, 33 I.

Französische und sächsische Gesandtschaftsberichte aus Dresden und Paris 1848-1849, i 59 .

Frauwallner, E., $45 \mathrm{I}$.

Frazier, E. F, $47^{2}$.

Freidel, F., 472.

Die freie Wohlfahrtspflege, 331.

Der Freiheitskampf des spanischen Volkes und dic internationale Solidarität, I 79 .

Freyre, G., 467/468.

Fried, M. H., 154/255.

Friedmann, G., 293/294.

Friedrich, C. J., 453.

Friedrichs, H., 33 I.

Frölich, P., 490/49r.

Froese, L., 18 I.

Les frontières européennes de I'U.R.S.S., 484/485.

Frye, R. N., 315.

Fryer, P., 507.

Fürsorge und Sozialreform, 168.

Fulford, R., 340.

Fuller, B., 466.

Gable, R. W., I 29.

Gablentz, O. H. v. d., 294, $453 / 454$.

Gaertringen, F. H. v., 496.

Gaitskell, H., 517.

Gall, A., 294/295.

The Gandhi Reader, 480.
Gandhi, M. K., $4^{80}$.

Garas, F., 49r.

Garaudy, R., 290.

Garthoff, R. L., 185 .

Gedat, G. A., I4I.

Gegen die Philosophie des

Verfalls, 290.

Genevès, J., 454.

Geoffre, F. de, 343/344.

Georges, C., 518 .

Gerscher, D. B., 299.

Gerson, L. L., 3 II $/ 3$ I2.

Geschichte des Zweiten Weltkrieges..., I 59 .

Ghosh, A., 480.

Gibb, H. A. R., 459/460.

Girard, A., I63/164, 488.

Gispen, W. H., 134 .

Gittler, J. B., I 5 I.

Gladden, E. N., $34^{\circ}$.

Glaeser, M. G., $47^{2}$.

Glick, P. C., 312.

Glick, Ph. M., 467.

Glum, F., 449.

Gneuss, Chr., I 26, 455 .

Godechot, J., 164.

Göhring, M., 158.

Görlitz, W., $331 / 332$.

Goetz-Girey, R., 454.

Gogol, N. V., 5II, 512/513.

Goguel, F., 490.

Gold, B., I 29.

Goldberg, A. J., 147.

Goldberg, H., 3 I I.

Goldfinger, N., 456/457.

Goldstein, A., 304/305.

Goldstein, I., I 47 .

Gollwitzer, H., I 26.

Goodman, L. L., 517.

Goriely, B., 484/485.

Goslinga, C. Ch., 143/144.

Gottwald, K., I 62 .

Gout, R., 134/135.

Graduate Employment, 340.

Granott, A., 482.

Granouillac, A., 326.

Graveson, R. H., $173 / 174$.

Green, C. McL., $472 / 473$.

Greenwood, G. A., 340.

Griffiths, P., 318.

Grimond, J., 506.

Grinten, W. C. L. v, d., 508.
Groener, W., 496.

Groenman, S., 517.

Groote, W. v., 497.

Groothoff, H. H., 455 .

Gros, P., 497.

Grottian, W., I81/182.

Gründer, K., 450.

Gruliow, L., 5 I I.

Grunebaum, G. E. v., 315 .

Guaita, M. L., 517 .

Günther, H. O., 318.

Günther, K. H., I75.

Guilbert, M., 164.

Guillain, R., 317.

Gulick, J., 320.

Gutzkow, K., 500 .

Haakman, A. J., 508.

Haas, E. B., 517.

Haber, W., 147 .

Habro, E., 479/480.

Haeuptner, G., 290.

Hagen, E. E., 477.

Hales, E. E. Y., 344.

Hallgarten, G. W. F., 460.

Halperin, E., $5 \times 5$.

Halsey, A. H., 339.

Halverson, P. M., 147/148.

Hamilton, E. C., I 34 .

Hammond, Th. T., sir.

Handbuch der Soziologie, 294.

Handcock, W. D., 339.

Harmand, G., 290/29I.

Harper, F. A., 517.

Hart, B. H. L., I38, I85.

Hartmann, G., 304.

Harvey, H., 506.

Hasluck, P., 5 I 7.

Hauptmann, G., 500 .

Hay, D., 46r.

Hayek, F. A., 13 I, 295.

Hazard, L., 456/457.

Hazlitt, H., I40/I4I.

Hegel, G. W. F., 29 I.

Heilbroner, R. L., 299/300.

Heine, H., 323/324/325, 497, 500 .

Heintz, P., 49x.

Heisenberg, W., 140.

Hellpach, W., 294.

Hentzen, C., 508/509. 
Herbermann, C., 336 .

Heriot, A., 508 .

Hersch, J., 294.

Hertel, G., 497.

Hertz, F., 497/498.

Herzen, A., I 82, 5 I 2/5 13 .

Herzfeld, H., 460 .

Herzl, Th., 300 .

Heuss, A., 498.

Heydte, F. A. Frhr. v. d., 294.

Hill, Ch., 459.

Hilton, R., 3 I8.

Hinterhäuset, H., 508 .

Hirsch-Weber, W., 498.

Hirschfeld, A., 49I.

Hirth, F., 497.

H. R. H. The Duke of Edinburgh's Study Conference..., $4 \sigma_{3}$.

History of Bulgaria, I6r.

Hock, W., 168.

Höhle, Th., 498.

Hoffmann, S., 491.

Hoffmeister, J., 29 r.

Hofland, H. J. A., 138.

Hofstätter, P. R., 517 .

Hogan, E. J., I 57.

Hogarth, Ch. P., 517.

Hoggart, R., 340/34I.

Holbach, P. H. D. d', 323/ $324 / 325$.

Holzgräber, R., 335/336.

Hoppla, wir leben!, 332.

Horowitz, I. L., I 26/1 27.

Hoselitz, B. F., 46I/462.

Hough, H. B., 473.

Houn, F. W., 479.

Hourdin, G., 326 .

Housing, Building and Planning, 304.

Howe, G., 126.

Hudson, G. F., 476.

Hünermann, W., 332.

Hugh-Jones, E. M., I 39.

Hughes, E., 341.

Hunold, A., 295.

Hunt, R. N. C., 5 I 2.

Hurtig, S., 49r.

Hutchinson, H. W., 3 Io.

Ibárruri, D., I 79.
Ickes, H. L., I 48.

Indian Communist Party

Documents, $480 / 481$.

Indien und Deutschland, 318.

Indonesia in 1956,517 .

Industrial Trade Associations, 503/504.

Inkeles, A., I82, 5 Io.

The Institute of Jewish Affairs Annual I956, 304/ 305 .

Les institutions sociales de la France, 326/327.

Integrity and Compromise, 29 I.

The sth International Conference on Planned $\mathrm{Pa}$ renthood, 463 .

Isambert-Jamati, V., I64.

Islam and the West, 3 I5.

Israel and Egypt, 517.

Itterzon, G. P. v., 134 .

Iyer, R., 476 .

Izard, G., 5 I 2.

Jack, H. A., 480 .

Jacoby, G., 304/305.

Jacquignon, L., 327.

Jahoda, M., I45.

Jahrbuch der D.D.R., 332.

Jahrbuch des Deutschen Genossenschaftsverbandes für 1954 , 1955 respectively $169,498 / 499$.

Janowitz, M., I 3 I.

Jászi, O., 300 .

Jaucourt, A. F. de, 323/324/ 325.

Jean-Baptiste, S.-V., 3 IO.

Jeandel, P., 484.

Jehring, J. J., 473 .

Jiggets, J. I., 3 I9.

Johannessen, E., 473 .

Johnson, F. E., I49.

Jones, D. B., I4.5.

Jong, J. J. de, I 59.

Joughin, J. T., I64.

Jouvenel, B. de, 454 .

Jurji, E. J., 476/477.

Kabes, V., 305 .
Kabir, H., I55.

Kägi, W., 295.

Kahane, E., 323/324/325.

Kahn, M., 517 .

Kaiser, B., I72, 503.

Kamenetsky, I., 5 I 2.

Kamnitzer, H., IGg.

Det Kampf der deutschen

Sozialdemokratie..., 499.

Kamphuisen, P. W., so9.

Karnik, V. B., 480/48I.

Kassalow, E. M., 456/457.

Kaussmann, E., I 40.

Kautsky, B., 500, 517 .

Kautsky, J. H., 48 I.

Kayfetz, B. G., 304/305.

Kayser, J., 494.

Kazakov, G., I 82.

Keiter, F., 294.

Kemp, B., 504.

Kennan, G. F., 346/347.

Kerr, C., 293, 456/457.

Kertesz, S. D., I 59.

Kettmann, H., 494/495.

Khan, M. Z., 3 I 5.

Khrushchev, N. S., 185 .

Khurshid, A. S., I 52.

Kiang, Lu-Yu, I 54.

King, J. K., 477.

Kirby, E. S., $478 / 479$.

Kirkbride, A. S., 477.

Klein, F., 459.

Klein, L. R., 293.

Klein, V., 296.

Kluckhohn, C., 5 Io.

Klutchevsky, B., s I 2.

Kluth, H., I67, 294.

Kluwer's zakboek sociale verzekering, 517 .

Kluxen, K., 34I.

Knaplund, P., 300 .

Knight, F., 504.

Koch, D. M. G., 482.

Koch, J., 494/495.

Köchling, A., $33^{6}$.

Kofler, L., 294.

Kohn, H., 295, 473/474, $512 / 5$ I3.

Kohr, L., 454.

Kolarz, W., I57, I82/183.

Komarovsky, M., 293.

Konkoly, K., 344 . 
Koontz, H., I 29.

Kooy, T. P. v. d., 129.

K. P. D.-Prozess, 169.

Kraan, K. J., 293.

Kracauer, S., 485.

Kramer, D., 474.

Kretzschmar, H., 159.

Kruck, A., 499 .

Krupp und die Hohenzollern, 499.

Krupskaja, N. K., 183.

Kuczynski, J., 332/333, 499.

Kühne, K., I 26 .

Kühner, O. H., IG9.

Kuhn, A., 305.

Kuin, P., 5 I 7 .

Kulski, W. W., 347 .

Kuper, L., 309.

Kyung Cho Chung, Is6/ I57.

Labin, S., 305 .

Lacour, R., 492.

Lacouture, J., $465 / 466$.

Lacouture, S., $465 / 466$.

Lafargue, L., I58, 32 .

Lafargue, P., I 58, 32 I.

Laidler, H. W., 474/475.

Lambotte, R., 517.

La Mettrie, J. O. de, 323/ 324/325.

Lampert, E., 5 I3.

Land Tenure, 463/464.

Landau, R., 308.

Landgrebe, L., 126.

Landmarks in the History of Physical Education, $300 / 301$.

Lange, M. G., 333, 335/336.

Langer, P., 346 .

Latoque, P., $326 / 327$.

Laugharne, G., 143 .

Lauterbach, A., 454/455.

Der lautlose Aufstand, 333 .

Lawrence, J., 513.

Lazarsfeld, P. F., 293.

Lazitch, B., 515 .

Le Bras, G., 327.

Lebret, L. J., 304 .

Lecercle, J. L., 323/324/325.

Leeuwen, C. v., 127.

Lefebvre, G., 323/324/325.
Le Goff, J., 30I.

Lehmann-Russbüldt, O., $305 / 306$.

Leifer, W., 315.

Lekachman, R, 293.

Lelonek, H., 335 .

Lengelle, M., 326 .

Lenin wie wir ihn kannten, 183.

Lenin, V. I., $183,333,347$, $348,511,512 / 513$.

Lens, S., $138 / 139$.

Leonhard, W., 184 .

Lepointe, G., 327 .

Lepp, I., $164 / 165$.

Lesire-Ogrel, H., 166.

Lester, R. A., 456/457.

Lettrich, J., I6I.

Leven, G., 319.

Levine, I. D., 183.

Levinson, H. M., I47.

Levy, R., 292.

Lewis, J. D., 300.

Leys, M. D. R., r65.

Lichtveld, L., 3 Io.

Lie, T., 464.

Lim Tay Boh, 518 .

Lindsay, J., I 74 .

Lindsay, J. O., 46r.

Lipset, S. M., 148, 293.

Litt, Th., 135, 295.

Little, E., 30r.

Livernash, E. R., 456/457.

Ljaskovskij, A., I 83/184.

Lloyd-Prichard, M. F., 134 .

Lochner, L. P., 135 .

Lockhart, R. H. B., 5 I3.

Löwenthal, F., 295 .

Löwith, K., 292.

Logan, H. A., 468.

Lohmar, U., I67.

Loosley, E. W., 468.

Lorenz, Ch., 294.

Loth, D., r48/r 49 .

Lowenthal, L., 293.

Lowenthal, M., 300 .

Lucas, H. S., 146.

Luck, H., 17 O.

Lucretius, 323/324/325.

Liuschow, H., 494/495.

Lukács, G., 291, 449, 450.

Lundberg, E., s09/510.
Lussu, E., $17^{6 .}$

Ly, A., 307.

McCance, R. A., I35.

Macgregor, G. H. C., 517 .

McIntosh, P. C., 300/301.

MacIver, R. M., 288, 291.

Maclean, F., 515/516.

McLean, H., SI 4 .

McManners, J., 490.

Mager, F., 333/334.

Maillet, J., 301 .

Mair, L. P., 142.

Maitron, J., $327 / 328$.

Malia, M. E., 514 .

Mammucari, G., 179.

Man's Role in Changing the

Face of the Earth, I 29/130

Manuel, F. E., 492.

Mao Tsé-Toung, I5s.

Marcel, M., 326.

Marcuse, H., 456 .

Mařik, J., 517.

Mark, B., 345 .

Markert, W., 295/296.

Markov, W., 493.

Marmier, J., 326.

Marmontel, J. F., 323/324/ 325.

Marshall, D., I 74.

Martin, F. M., 339.

Martin, A. v., 130 .

Martz, J. D., 3 Io.

Marx, K., I 70 , 334, 334/335, 500.

Marxismus-Studien, 455.

Les Marxistes répondent à leurs critiques catholiques 517.

Masani, R. P., 48 I.

Maschkin, N. A., 301 .

Masini, P. C., 344/345.

Maspétiol, R., 460.

Masse und Demokratie, 295.

Massin, J., 165.

Materialien zur Feststellung der wirtschaftlichen und sozialen Lage der Landwirtschaft..., 500 .

Matthias, E., 455 .

Maung, M., 153.

Maus, H., 294. 
Maxwell, R. S., 474.

Mayer, H., 500 .

The Measurement and $\mathrm{Be}-$ havior of Unemployment, 295.

Meder, W., $34^{8 .}$

Mehnert, K., I53.

Mehring, F., 450, 500.

Meier, P. J., 516.

Meier, R. M., I 3 .

Meister, A., 492.

Meisterwerke deutscher Literaturkritik, 500 .

Memmi, A., 464.

Mendieta Y Nunez, L., 455 .

Der Mensch im kommunistischen System, 295/296.

Mensching, G., 294.

Metidor, Y., 482.

Merleau-Ponty, M., 292.

Merlino, S., 344/345.

Merton, R. K., 455/456.

Metzke, E., 295/296, 455 .

Meulen, D. v. d., $483 / 4^{8} 4$.

Meutey, P., I63/164.

Meyer, A. G., 513 .

Meijerink, G., 178,517 .

Michael, F. H., 315.

Michel, E., 30I.

Michels, T., 477.

Miesbach, H., 173.

Mikat, P., 348.

Milhau, J., $4^{88 .}$

Mills, C. W., 149.

Minney, R. J., I55.

Mises, L. v., 296.

Misra, B. R., 3I8/3I9.

Moch, J., I 84.

Modigliani, G. E., 507.

Molen, G. H. J. v. d., I34.

Mondolfo, R., 507.

Monsterleet, J., I55.

Montagu, I., 320/32I.

Montgomery, J., 34 r.

Moore, S. W., 296.

Moraes, F., 517.

Morais, H. M., $469 / 470$.

Morazé, Ch., i 58.

Moreell, B., 474.

Morelly, 323/324/325.

Mori, G., 508 .

Morsa, J., $4^{87}$.
Mosely, Ph., SI4.

Mosher, A. T., 309 .

Moudry, V., 485 .

Mounier et sa génération, 492.

Mounier, E., 492.

Mounier-Leclercq, P., 492.

Mühlpfordt, G., 32 I.

Mueller, I. W., 174/175.

Müller, K. V., I 30.

Muhs, K., 294.

Mukherjee, R., 309.

Mumford, L., I 29/130.

Munby, D. L., I 27.

Munrow, A. D., 300/301.

Myers, J., 474/475.

Myrdal, A., 296.

Myrdal, G., 456 .

$\mathrm{Na}$ obranu republiky proti fašismu a válce, 162 .

Naas, B. G., I42.

Nadel, S. F., 130/131.

Nagy, B., 344 .

Nantet, J., 301/302.

Nationalism and Progress in Free Asia, 3 I $5 / 316$.

Naunin, H., 336 .

Naville, P., 492/493.

Neale, J. E., 34I.

Nearing, H., 475.

Nearing, S., 475 .

Nehru, J., 480.

Nell-Breuning, O. v., 126, I 27, 456 .

Nemec, F., 485 .

Neumann, F., 456 .

Neumann, F. W., 348.

Neumark, S. D., 466.

Neundörfer, L., 5 I 8 .

Neuss, E., 500/50I.

The New Cambridge Modern History, $46 \mathrm{r}$.

New Concepts in Wage

Determination, $456 / 457$.

Niemeyer, G., I 84 .

Niemöller, W., 170.

Nkrumah, K., 308.

Les noirs dans le monde, 518.

Noland, A., 165.

Nove, A., I 84 .
Der Nürnberger Prozess, soI.

Nümberger, R., 455 .

Nuseibeh, H. Z., 316.

Oakeshott, M., 295 .

Obermann, E., 335.

Obermann, K., 459 .

O'Connor, H., 302.

Die öffentliche Fürsorge, 335 .

Ollenhauer in der Karikatur, 335 .

On Freedom and Frec Enterprise, I 3 I.

Origo, I., 345.

Ormel, D. W., 293.

Ormeling, F. J., I 56 .

Oser, J., I39.

Osmańczyk, E. J., 179.

Osterkamp, K., 335 .

Owen, F., ${ }_{4}^{67}$.

Owen, R., 175 .

Pankhurst, R. K. P., 504 .

Parker, F. E., 3 I 2.

Parsons, K. H., 463/464.

Parsons, T., 296/297.

Parteien in der Bundesrepublik, 335/336.

Pasteur, L., 323/324/325.

Peace in the Middle East, 518.

Peacock, A. T., 506.

Pear, T. H., 303.

Pearson, H. W., 302.

Pelling, H., 504/505.

Penn, R. J., 463/464.

Peters, H. M., 294 .

Peterson, H. C., 475.

Petro, S., 475.

Petrov, E., 348 .

Petrov, V., 348.

Petry, R. C., 450.

Petz, R., 158.

Pfeiffer, G., 169.

Philip, A., 493.

Phillips, W. T., 315/316.

Les philosophes célèbres, 292.

Pierre, J., 323/324/325.

Pierson, F. C., $456 / 457$. 
Piettre, A., 304, 457.

Pisky, F., 322.

Planck, R. E., 293.

Plekhanov, G. W. (N. Ramsaur Jr., E. E., sıo.

Beltow), 348, 450 .

Pocock, D., 5 I6.

Pocock, J. G. A., 505.

Podossetnik, W. M., 450/ $45 \mathrm{I}$.

Poggi, A., 507.

Polanyi, K., 302.

Political Behavior, 13 I.

Political Economy, 457.

Pollard, H. M., I 59/160.

Pollock, F., 139, 306.

Polonsky, A., 3 I 2.

Ponting, K. G., 505 .

Poperen, J., 323/324/325.

Population Theory and Policy, I3 I.

Postgate, R., I35/136, 341/ 342.

Potter, G. R., $46 \mathrm{I}$.

Poulain, A., $303 / 304$.

Pour un bilan de la formation ouvrière, 485 .

Pouthas, Ch. H., 165/166.

Prakke, H. J., 518 .

Prasad, R., 48r.

A Preface to the Social Sciences, 457.

Price, H. D., 475 .

Priebe, $H_{\text {., }} 494 / 495$.

Problems of the Malayan Economy, 5 I8.

Procacci, G., 177.

Le progrès au service de l'homme, I66.

Proudfoot, M. J., 160.

Psichari, H., 323/324/325.

Pucheu, R., 326.

Pulec, V., 149.

The Push-Button World, 139.

Putlitz, W. G. E. Herr Zu, 170.

Pyziur, E., I 32.

Querido, A., 345 .

Quesnay, F., 323/324/325.

Quigley, H. S., 483.

Quilitzsch, S., 32 I.
Rachlin, C., 312.

Radaceanu, L., 5 I8.

Ramm, Th., 455.

Randwijk, H. M. v., 477.

Rauch, G. v., 348, 5 I4.

Raup, Ph. M., 463/464.

Read, L. E., I 38 , I 49 .

Redmayne, P., 308.

Reed, H. A., 3 I 5 .

Rehbein, G., 334 .

Reich, H., I4I.

Der Reichsfreiherr vom und zum Stein und die westfälisch-lippische Selbstverwaltung, 336 .

Reigrotzki, E., r70/r71.

Reiss Jr., A. J., I45/I46.

Relgis, E., 5 I 8.

Religion and Social Work, I49.

Reshetar Jr., J. S., 184 .

Rewolle, H. D., 173.

Rewriting Russian History, I 80.

Reynolds, L. G., 456/457.

Richter, M., I7I, 338, 502.

Rickett, Adele, 479 .

Rickett, Allyn, 479 .

Riedmatten, L. de, $46 \mathrm{r}$.

Riessen, H. v., 293.

Rimscha, H. v., 348.

Riou, M., 5 I8.

Ritter, J., 450.

Robespierre, M., 323/324/ 325.

Robinson, N., 306.

Robson, R., 342.

Rocard, M., I66.

Roeder, B., 348.

Röhrs, H., I 27/I 28.

Röpke, W., I3I, 295.

Rössler, H., I 7 I.

Rössler, R., 348.

Rogers, G., 175.

Rohlfing, T., I 73 .

Romulo, C. P., 464 .

Roos, H., 345 .

Roos, F. de, 293.

Rose, S., 476.

Rosenberg, A., 336.

Rosenberg, B., 312/313.
Rosenberg, H., 292.

Rosental, M., 336.

Ross, A. M., 456/457.

Rossi, A., 514.

Rostow, W. W., 479.

Rothbaum, M., 456/457.

Rougemont, D. de, SI4.

Rousseau, J. J., 323/324/

325.

Rousseaux, P., 454

Roy, C., 308/309.

Royer, J. M., 49 I.

Rubel, M., sor.

Ruben, W., 45 I, 481.

Rueff, J., I3 I.

Rühle, J., 457/458.

Rüstow, A., 295 .

Rumage, Ch. J., g 16.

Russian Thought and

Politics, 5 I 4 .

Rustow, D. A., 3 I 5.

Rutkewitsch, M. N., 45o/ 451.

Ryan, A. P., 342.

Sachs, E. S., 466.

Sadat, A. El, 307 .

Sahmer, H., I73.

Saint-Just, A. L. L. de, $323 / 324 / 325$.

Saint-Maurice, H., $45^{8}$.

Saint-Simon, H. de, 323/ 324/325.

Sakr, C. S., 142.

Salem, E. A., 292.

Salin, E., 140.

Salomon, A., I 32.

Salvemini, G., 507 .

Die Sansculotten von Paris, 493.

Sauer, C. O., $129 / 130$.

Sauvy, A., 304.

SBZ von 1945 bis 1954,171 .

Schachtner, R., $336 / 337$.

Schäfer, Wilhelm, 337 .

Schäfer, Wolfgang, 50r.

Schapiro, L., I85.

Scheibert, P., 126.

Scheibner, O., I 32 .

Scheler, H., 306.

Schelsky, H., I67.

Schiavi, A., 507. 
Das Schicksal der Deutschen in Ungarn, 507.

Schlechte, H., I 59, 494.

Schlesier, E., 297.

Schmeer, K., , 01 .

Schmid, C., 516.

Schmid, P., I 53.

Schmidt, J., 329.

Schneider, B. V. H., I so.

Schneider, W. M., 3 I3.

Schneider-Henn, D., 176.

Schoeck, H., 295.

Schöffer, I., $160, x 78$.

Schoell, F. L., 309.

Schraepler, E., 501/502.

Schram, S. R., 484/485.

Schran, P., 498.

Schubert, E., 494/495.

Schütz, K., 335/336, $49^{8}$.

Schuffenhauer, W., 290.

Schulte Nordholt, J. W., I 50.

Schultz, J., 337.

Schultz, Th. W., 142.

Schulz, G., 335/336.

Schulz, K. P., I 7 I.

Schwarzenberger, G., 297.

Scott, E. J., 184.

Seear, N., 506.

Seeley, J. R., 468.

Sennholz, M., I 3 r.

Seraphim, H. G., 336.

Seraphim, H. J., $4^{86 .}$

Sergot, A., 305 .

Shabad, Th., 3 I 7 .

Sharp, M. P., 313 .

Shenfield, B. E., 505 .

Shepherd, G., 487.

Shepperson, W. S., 505/506.

Sherman, G., 184 .

Shister, J., 518.

Shuchman, A., 337 .

Shumsky, A., 482.

Siebrecht, V., 502.

Sieburg, F., I66.

Sieburg, H. O., $485 / 486$.

Siedlung und innere Kolonisation im europäischen Raum, 486.

Siegel, A., I 50.

Siegfried, A., 166 .

Siemsen, A., 338 .
Silberner, E., I60, 458.

Silone, A., 507.

Sim, R. A., 468.

Simon, F., 493.

Simon, H. A., 297.

Simon, P. H., 518.

Sindler, A. P., 476 .

Size and Morale, 518.

Skendi, S., 322.

Slusser, R. M., 5 I4.

Smelser, N. J., 296/297.

Smet, R. E. de, 322/323.

Smirin, M. M., 459.

Smit, M. C., I34.

Smith Jr., C. J., I 84 .

Smith, H. P., I75.

Smith, L. E., I43.

Smith, W. O. L., 306.

Smitskamp, H., 134 .

Soboul, A., 323/324/325, 459,493 .

Sociaal-wetenschappelijke verkenningen, 509 .

Social and Political Thought in Byzantium, 46r.

Social Implications of Industrialization and Urbanization in Africa South of the Sahara, 465.

Social Welfare and the Citizen, 518.

Somerville, J., Iso.

Soustelle, J., 328.

Soviet Affairs, 184 .

The Soviet Army, 185 .

The Soviet Secret Police, SI4.

Das Sowjetsystem in der heutigen Welt, 348 .

Soziale Betriebs-Praxis, 5 I 8.

Soziale Sicherung auf dem Lande, 493.

Sozialplan für Deutschland, 502.

Die Sozialreform, 171, 338, 502.

Spelt, J., I43.

Spengler, J. J., I3 3 .

Stammer, O., 294.

Stanley, T. W., 3I3.

Starlinger, W., I39/ 4 40, $306 / 307,514 / 515$.
Starobinski, J., 292.

State Workmen's Compensation Laws as of September I954, 5 I 8 .

Statistisches Jahrbuch der D.D.R. $1955,171 / 172$.

Staugaard, W., 464.

Steiniger, P. A., jor.

Stern, F., I 36.

Stern, L., 499, 5 I 8.

Stemberger, D., 332.

Stierli, J., I 54.

Storry, R., 483.

Strayer, J. R., 293.

Streisand, J., 459.

Stremecki, J., 321/322.

Strickert, H. G., I69.

Sturmthal, A., 303.

Südosteuropa-

Bibliographie, 161 .

Swan, M., 468.

Swearingen, R., 346.

Sweet-Escott, B., 5 I 8.

Sweezy, P. M., $297 / 298$.

Szczepanik, E. F., 478/479.

Szelpal, A., I 40.

Tang, P. S. H., 479.

Tanner, V., 488.

Tartler, R., 167.

Tasca, A., 518.

Taylor, F. J., I so.

Taylor, G. E., 315 .

Taylor, G. W., 456/457.

Taylor, T., I 50.

Tersen, E., 493/494.

Texcier, J., S 18.

Texte der indischen Philosophie, $45 \mathrm{I}$.

Textes choisis de 1" "Encyclopédie", 323/324/325.

Thapar, R., 319.

Thayer, Ph. W., 3I5/3I6.

The Theory of Wage Determination, 298.

Thieding, F., I 7 I.

Thielicke, H., I 26.

Thieme, K., 348.

Thier, E., 455 .

Thomas, D. L., 483.

Thomas, W., $45 \mathrm{I}$.

Thomas Jr., W. L., I 29/I 30. 
Thompson, A. F., 490 .

Thompson, F., ISI.

Thompson, L., 307.

Thomson, E., 486.

Thorez, M., 328.

Thorne, W. J., 342.

Thursby, V. V., 457 .

Thys, W., 178 .

Tiano, A., 166 .

Timbal, P. C., 328.

Tinker, H., 316 .

Tisserand, M., 323/324/325.

Töpfer, B., 5 I8.

Tokaev, G. A., 349.

Tomasic, D. A., 321/322.

Tootell, G., 293.

Torr, D., 175 .

Touchard, J., 490.

Toynbee, A., 302.

Toynbee, V. M., 302.

Trachtenberg, A., I48.

Trade and Market in the

Early Empires, 302.

Trager, F. N., I 54 .

Treadgold, D. W., $5 \times 4$.

Treue, W., 502.

Triesch, G., I 72.

Trofimov, P. S., 290.

Tropp, A., 342/343.

Trow, M., 148, 293.

Turgot, A. R. J., 323/324/ 325.

Turner, J. E., 483.

Ubersfeld, A., 323/324/325.

Die UdSSR in Zahlen, 349 .

Übersicht über die Bestände des Deutschen Zentralarchivs Potsdam, 502.

Uhlan, E., 483.

Uhlig, H., 172 .

Understanding Minority

Groups, $15 r$.

Unnik, W. C. v., $\mathrm{I} 34$.

The Unservile State, 506.

Unterberger, B. M., 3I3/ 3 I 4 .

Utz, A. F., 458 .

Valiani, L., s 58.

Valjavec, F., I6I.

Valko, L., 322.
Varain, H. J., 338.

The Varieties of History, I 36.

Varloot, J., 323/324/325.

Veldkamp, G. M. J., 178/ i79.

Venturini, A., 344/345.

Vermeulen, E. E. G., I 36 .

Vers la promotion de l'économie indigène, $4^{65}$.

Verwey-Jonker, H., 509 .

Vexliard, A., 298.

Viau, P., 326 .

Vickrey, W. S., 293.

Vierhaus, R., I 36 .

Vignaux, P., 325 .

Villard, H. S., 308.

Villey, M., 292/293.

Vincent, P., 484.

Virchow, M., 335/336, 498 .

Vlekke, B. H. M., 517.

Vokopola, Q., 322.

Volguine, V. P., 323/324/ 325.

Volin, I85.

Voltaire, $323 / 324 / 325$.

Wagner, G., 142.

Wallace-Hadrill, J. M., 490.

Waller, R. D., I 74.

The War and the Neutrals, 302.

Warrender, H., 343.

Warriner, D., 3 I6.

Watson, G., s06.

Wearmouth, R. F., 506.

Weaver, H. G., 128.

Weber, M., I 32.

Webster, D., 138.

Weerth, G., 172, 503 .

Der Weg zum industriellen Spitzenverband, $172 / 173$.

Weidlé, W., 185 .

Weinstock, H., I33.

Weis, E., 328.

Weise, H., 343 .

Weisenborn, G., 333.

Weiss, G., 294.

Weissberg, A., 486.

Weisser, G., 294.

Wendland, H. D., 126.

Wesemann, F., 173.
Wetter, G. A., 1 26, 349.

Wheeler, G., 184 .

Wheeler, H., 518.

Wheeler-Bennett, J. W., 486.

Whitaker, A. P., 142/143.

White, R. J., 506.

Whyte Jr., W. H., $47^{6}$.

Widdowson, E. M., I35.

Wiese, L. v., $133,318$.

Wilensky, H. I., 3 I 4 .

Willard, C., 323/324/325.

Willard, G., 323/324/325.

Willetts, R. F., 300/301.

Williams, L. F. R., 48z/483.

Williams, P. M., 490.

Williams, W. A., 3 I r.

Wilson, Ch., $486 / 487$.

Wilson, P., s 8 .

Winckelmann, J., I32.

Winiger, A., $14^{\circ}$.

Winnick, L., 314.

Winter, E., 322.

Wirsing, G., 301.

Wischnitzer, M., 3 I 4 .

Wittfogel, K. A., 298.

Wittke, C., $314 / 315$.

Die WK-Reihe, 173 .

Wohlgemuth, P., 154 .

Wolfe, B. D., 185 .

Wolin, S., 514.

Wood, A., so6.

Wootton, G., 176 .

A World on the Move, 477.

Wormuth, F. D., 469.

Wright, M. C., 317.

Wright, R., I40.

Wucher, A., 136/137.

Wyschinski, A. J., 185/186.

Yalman, A. E., 157.

Yarmolinsky, A., s I5.

Yershov, P., I 86.

York, R. M., I I.

Young, A. F., 176.

Young, G. M., 339 .

Young, S. G., 457.

Youtsler, J. S., I 5 I.

Yuan-Li Wu, 317/318.

Za chléb, práci, pudu a svobodu, I62. 
Za svobodu českého a Ziegenfuss, W., 294. slovenského národa, 162. Zaar, I., 156.

Zaleski, E., I 86.

Zaleznik, A., 298/299.

Zangheri, R., 507.

Ziebill, O., 338 .
Zuhorn, K., 336.

Zuidema, S. U., 293.

Zur Ökonomik und Technik der Atomzeit, $x$ 4o.

Zweiling, K., 5 I 8 .

Zwilgmeyer, F., 294 . 